\title{
Antibiotic resistance profiling of Uropathogenic Enterobacteriaceae, Casablanca, Morocco
}

\section{Aniba Rafika, Barguigua Abouddihaja, Dihmane Asmaaa, Nayme Kaotarb, Timinouni Mohammed ${ }^{b}$.}

${ }^{a}$ Laboratory of Biotechnology and Sustainable Development of Natural Resources, Polydisciplinary Faculty, Sultan Moulay Slimane University, Beni Mellal, Morocco.

${ }^{\mathrm{b}}$ Molecular Bacteriology Laboratory, Institut Pasteur de Maroc, Casablanca, Morocco.

\begin{abstract}
.
Introduction: the urinary tract infection is a pathology frequents as well in community as at the hospital. In last years, there has been increased the incidence of antibiotic resistance in Uropathogenic Enterobacteriaceae. This development explains the need for periodic regional surveillance of this resistance to antibiotics, which poses a real problem of public health, because it affects several molecules ranging from $\beta$-lactam to quinolones.
\end{abstract}

Objective: the aims of this study is to determine the profile of antibiotic resistance of Uropathogenic Enterobacteriaceae isolated from medical analysis laboratories in Casablanca, Morocco.

\begin{abstract}
Materials and methods: This is a prospective study that was conducted on patients who visited medical analysis laboratories in Casablanca city, for urinalysis test from 01 January 2017 to 30 December 2018 (a two full years). The culture was performed according to the usual techniques, and the antibiogram was done according to the recommendations of the Antibiogram Committee of the French Society of Microbiology. The statistical analysis was performed using Microsoft Excel (Microsoft 2016).
\end{abstract}

Results: During our study, we reported $18 \%$ of urinary tract infections, Sex ratio F/M was 1.79 . In addition, Enterobacteriaceae were the most common germs. Of all the strains isolated, we noted a predominance of Escherichia coli with $75.41 \%$ ) and Klebsiella ssp with $17.05 \%$.

In the course of this study period, we observed high rates of resistance to the main classes of antibiotics, and an overall increase between 2017 and 2018 in this resistance to the majority of $\beta$-lactam antibiotics, quinolones and aminoglycosides for almost all species of Uropathogenic Enterobacteriaceae. However, amikacin was the most active molecule against the Uropathogenic Enterobacteriaceae strains isolates.

Conclusion: The development of resistance of Uropathogenic Enterobacteriaceae to antibiotics is a worrying phenomenon that exposes us to difficulties in the therapeutic management of infections. The current control of antimicrobial resistance is a real emergency and requires the involvement of public awareness before government instructions.

Key Words: Urinary tract infection, Cytobacteriological Urine Examination (ECBU), Enterobacteriaceae, antibiogram, antibiotic resistance.

\section{Introduction}

Urinary tract infections (UTIs) are a severe public health problem and are caused by a range of pathogens, but most commonly by Enterobacteriaceae family: Escherichia coli, Klebsiella spp, Proteus spp, Enterobacter spp [1]. According to the world health organization "WHO", urinary Enterobacteriaceae infection is one of the most common infections acquired in community and hospital [2].

The routine therapy of UTIs is based on the use of antibiotics such as $\beta$-lactams, quinolones and aminoglycosides in many countries. Unfortunately, the widespread and misuse of these antibiotics resulted in the increasing rate of resistance to them in the societies [3].

In the last years, the increase in rates of antibiotic resistance among Enterobacteriaceae has posed challenges in choosing regimens, especially when infection due to multidrug resistant Enterobacteriaceae are suspected or endemic. Also, it's become a major international public health issue, raising fears of therapeutic impasses, that's why it has the priority for surveillance and resistance studies given their high frequency and severity $[\mathbf{4}, \mathbf{5}]$.

In Morocco, the data on resistance to antibiotics of Uropathogenic Enterobacteriaceae are very limited and needs further investigations [6, 7].

Therefore, the global purpose of our study is to describe the antibiotic resistance profiling of Uropathogenic Enterobacteriaceae strains isolated at medical analysis laboratories in Casablanca, Morocco, in order to supervise their emergence and to restrict the spread in the population.

\footnotetext{
* Corresponding author: RAFIKANIBA1466@gmail.com
} 


\section{Materials and methods}

\section{Setting}

This study was designed as a point-prevalence survey performed between 01 January 2017 and 31 December 2018, it was conducted in medical analysis laboratories, Casablanca, Morocco. Casablanca is the economical capital of Morocco and the largest city in country.

The activities of those laboratories consist in carrying out medical analyzes on behalf of private clients, clinics and hospitals, it admitted patient from all specialties and all ages from all regions of Morocco.

\section{Study population and data Collection}

2090 urines samples were collected throughout Casablanca from the health facilities, for each patient who came to the laboratory for a cytobacteriological examination of urines, a sterile sampling tube was given.

Patients were assisted with strict hygiene measures to ensure an aseptic sample. Prior to the collection, a survey from was made available to capture patient's details, these were related to personal and medical information.

\section{Collection and identification of strains}

The biologic criteria of inclusion were a pure bacterial culture with a colony count $\geq 105 \mathrm{CFU} / \mathrm{ml}$ associated with a leukocytes count $\geq 104 / \mathrm{ml}$. Culture was performed by inoculating $10 \mathrm{~mL}$ of urine onto CLED (Cystine Lactose Electrolyt Deficien) agar. After incubation at $37^{\circ} \mathrm{C}$ aerobically overnight, bacteria were identified using routine microbiologic procedures including biochemical test (API 10E or 20E system-bioMerieux $\AA$, Marcy l'Etoile, France and Vitex 2 compact 15 systembioMerieux $($ ).

\section{Antimicrobial drug susceptibility testing}

The study of antibiotic susceptibility was carried out by the Muller Hinton agar (Bio-Rad, Marnes-la-Coquette, France) diffusion technique and VITEK 2 compact 15 system bioMerieux ${ }^{\circledR}$ cards. Following overnight incubation, the inhibition zone diameters were measured, each strain was classified as susceptible (S), intermediate (I) or resistant (R) with interpretative reading according to the recommendations of the antibiogram committee of the French Microbiology Society (CA-SFM) [8].

All Uropathogenic Enterobacteriaceae strains were subjected to antimicrobial susceptibility testing against five antibiotic classes: Penicillin, cephalosporin, fluoroquinolones, aminoglycosides and folate pathway inhibitors (Trimethoprim-sulfamethoxazole).

\section{Statistical analysis}

Data were analyzed using the statistical software Excel, Microsoft 2016. This data included the identification number, the patient's name, the date of isolation, the germ identified, as well as the antibiotics tested with their susceptibility profile (S, I, R).

Categorial variable were expressed as percentages, and continuous variable were expressed as means \pm SD or median or median. Prevalence of infected patients were calculated.

\section{Results}

\section{Patient characteristics}

During the study period, 2090 urine samples were collected, included 374 infected patients had urinary tract infection (18\% of positivity).

The average age of patients infected in our series was $34.77 \pm$ years old with extremes age ranging from 1 month to 97 years.

The most common symptoms observed among patients infected are fever and urinary and kidney problems. Urinary cytology showed erythrocytes, polynuclear neutrophils infiltrates, epithelial cells, yeasts and urinary crystals, of which calcium oxalate crystals were the most numerous.

The female sex was predominant with $64.17 \%$ female compared to $35.83 \%$ male, with a F/M sex ratio of 1.79 (240 females and 134 males patient).

\section{Prevalence and pathogens of infections}

We isolated 381 bacteria with $306 \quad(80,31 \%)$ Enterobacteriaceae, $35(9,19 \%)$ Non fermenting Gramnegative Bacilli ferment and 40 (10,5\%) Gram positive cocci.

There were 306 non-duplicate Enterobacteriaceae isolates recovered from the infected patients (some of these patients had UTIs caused by more than one organism in the same episode).

The majority of uropathogens were Escherichia coli with $72 \%$ (230 strains, followed by Klebsiella spp (17.05\%). On the other hand, the other Uropathogenic species (Enterobacter spp, Proteus spp) were also isolated but with very low proportion. The distribution of the bacteria in patients with UTIs is shown in Figure 1.

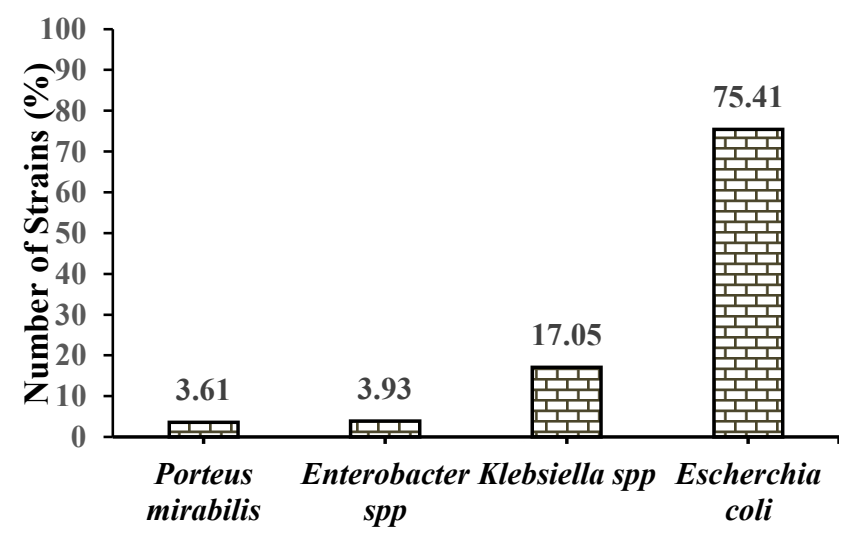


Figure 1 : The frequency of distribution of isolated bacterial strains

Antibiotic Resistance of Uropathogenic Enterobacteriaceae

In the course of our study, we sought to determine and interpret the profiling of Uropathogenic Enterobacteriaceae to various antibiotics tested according to CASFM standards.

Profile and development of $\beta$-lactams antibiotics resistance

The antibiotic resistance profiling of the different Uropathogenic germs shows a high resistance rate of $E$. coli for ampicillin (63.16\%), Ticarcilin (57.87). As well as, for the amoxicillin-clavulanic acid combination $(38.16 \%)$. The resistance rate of E. coli strains to 3rd generation cephalosporin was $16.9 \%$ and $16.96 \%$ for Cefoxitin (2rd generation cephalosporin).

With regard to Klebsiella spp strains, resistance was representatively of $53.84 \%, 46.77 \%, 39.22 \%$ for the combination of Amoxicillin-Clavulanic acid, 3rd generation cephalosporin and Cefoxitin.

In addition, Enterobacter spp had significant resistance to $\beta$-lactams with percentages of $76.72 \%, 61.54 \%$ respectively for the combination of AmoxicillinClavulanic acid and 3rd generation cephalosporin.

We also noted that $77.77 \%$ of Proteus spp strains are resistant to the combination of Amoxicillin-Clavulanic acid. While cephalosporin remains the most active antibiotics on these strains.

Rate of $\beta$-lactams antibiotics resistance for Enterobacteriaceae summarized in Figure 2 and $\mathbf{3}$.

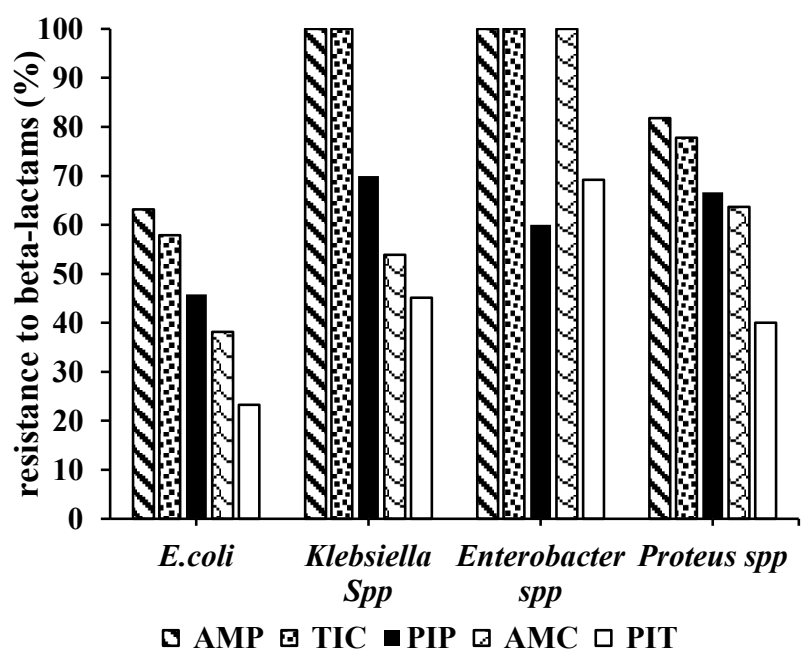

Figure 2 : Beta-lactam (penicillin) resistance profile, of uropathogenic isolates $(\%)$

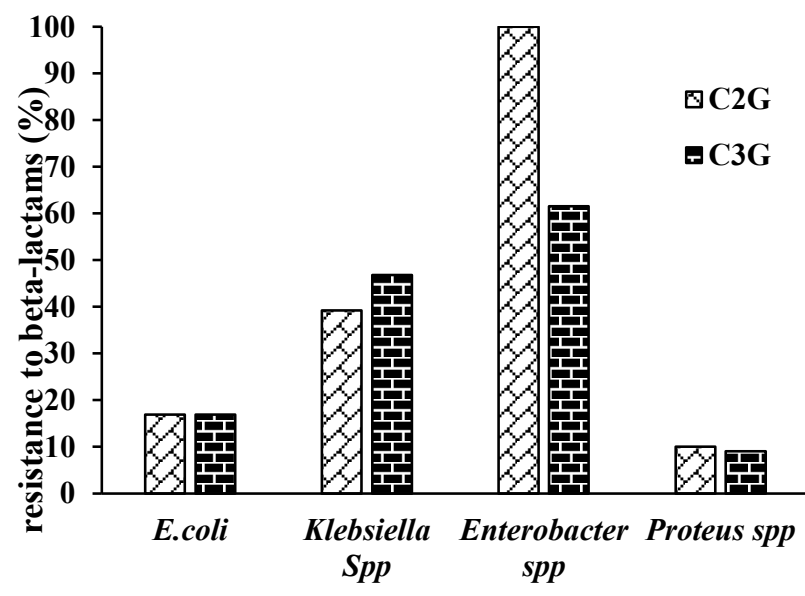

Figure 3 Beta-lactam (cephalosporin) resistance profiling of uropathogenic isolates (\%)

Profile and development of aminoglycosides antibiotic resistance

For E. coli strains, we recorded $27.85 \%$ resistance for Tobramycin and $22.93 \%$ for Gentamicin. we also noted that Klebsielle ssp strains register a moderate resistance to aminoglycosides which reaches a prevalence of $42.31 \%$ to Tobramycin and $38.46 \%$ to Gentamicin. Whereas, Enterobacter ssp strains showed significant resistance to Tobramycin and Gentamicin (61.54\%). The level of resistance of Proteus spp to aminoglycosides is $27.27 \%$ for Tobramycin and Gentamycin. Amikacin retains good activity against strains of Uropathogenic Enterobacteriaceae.

Rate of aminoglycosides antibiotics resistance for Enterobacteriaceae summarized in Figure 4.

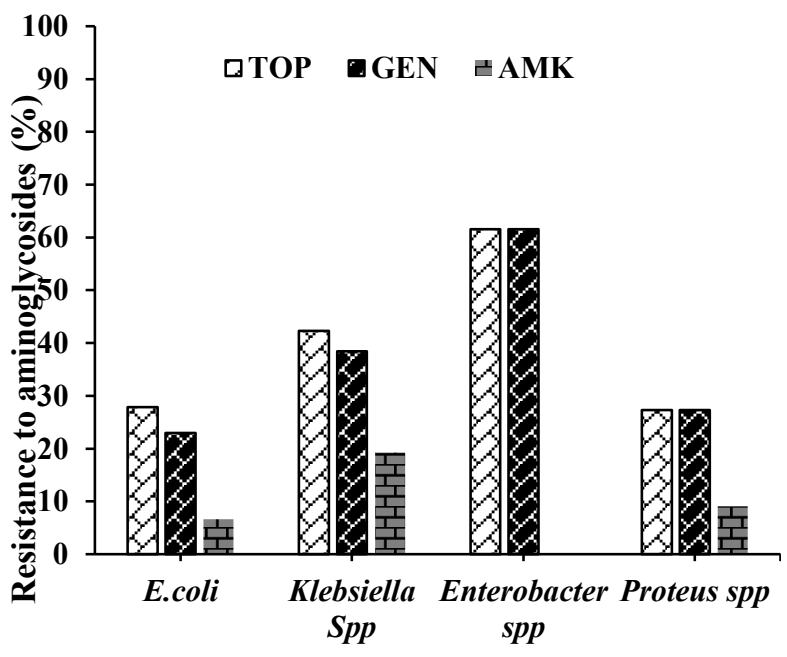

Figure 4 : Aminoglycosides resistance profiling of uropathogenic isolates $(\%)$

Profile and development of Quinolones antibiotic resistance

E. coli strains have mild resistance to Quinolones (Ofloxacin 27.19\%, Norfloxacin 27.78\%, Ciprofloxacin $26.52 \%)$. With regard to Klebsielle ssp strains, we noted 
$46.94 \%$ of resistance for Norfloxacin, $50 \%$ for Ofloxacin and 43.14 for ciprofloxacin.

In addition, quinolones resistance in Enterobacter spp strains has been identified with $50 \%$ of strains resistant to Ofloxacin, $46.15 \%$ of strains resistant to ciprofloxacin and $36.36 \%$ of strains resistant to Norfloxacin. However, $18.18 \%$ of Proteus spp strains are resistant to all fluoroquinolones.

Rate of quinolones antibiotics resistance for Enterobacteriaceae summarized in Figure 5.

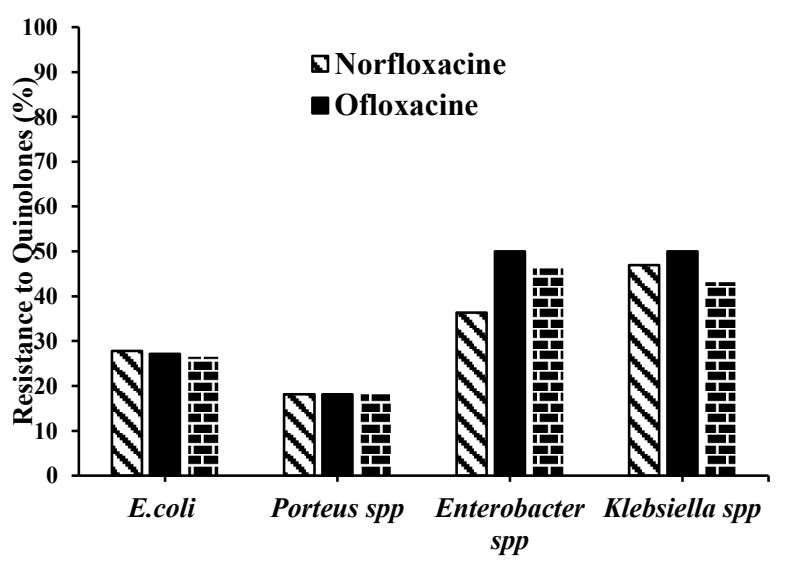

Figure 5 : Quinolone resistance profiling of uropathogenic isolates $(\%)$.

\section{Discussion}

Antibiotic resistance of bacteria a serious threat to global public health. The spread of resistant bacteria leads to a dramatic increase in mortality, morbidity and the cost of treatment [5].

It's an increasingly serious threat to global public health that requires action across all government sectors and society [9].

Among the germs responsible for urinary tract infections, Enterobacteriaceae are the most formidable, because they have mechanisms of resistance to many antibiotics, the high concentration of these germs in the digestive tract, promotes the exchange and dissemination of resistance genes [10].

Thus, for our study with a sample of 2090 patients, we obtained 1716 negative urine cultures (82.11\%) and 374 positive cultures, giving a frequency of urinary tract infection of $17.889 \%$. This positivity rate is slightly lower than that obtained in subsequent studies by Hailaji et al., 2016 in Nouakchott city, Mauritania $(18,4 \%)$ [4], and a slightly higher than that found by Mahmoud et al ., 2010 in Fes city, Morocco [10] and Lahlou et al., 2009 in Meknes city, Morocco [11]. who obtained positive urine, and culture rates of $14 \%$ and $12,2 \%$, respectively. This situation is justified by the fact that urinary tract infection is one of the most frequent community bacterial infections [12]. The negative urine culture rate represents more than three-quarters of all the examinations carried out in our study. This can be explained by the fact that these patients are subjected to self-medication without medical consultation, which contributes to hide the pathogenic bacterial flora and hinders its multiplication on the culture media in the laboratory [13].

The high frequency of urinary tract infections in women compared to that in men is consistent with that reported in the literature review [13, 4]. In infected patients on our study, 63,17 \% were women, whereas $35.83 \%$ were men. This is explained by the fact that urinary tract infection in women can be interpreted by several factors related to the anatomical and physiological nature of their urinary tract since the length of their urethra is much reduced $(4 \mathrm{~cm})$. Also, hormonal and physiological changes can promote the occurrence of these infections $[13,4,14]$. Man is relatively more protected because of the anatomical structure of his urinary system. Fecal contamination is then reduced since there is a distance between his anus and his urinary meat $[\mathbf{1 3}, \mathbf{4}]$.

The ascending pathophysiology of urinary tract infection and the strong colonization of the perineum by Enterobacteriaceae of digestive origin, and in particular Escherichia coli, associated with specific factors of uropathogenicity such as bacterial adhesins capable of binding to the urinary epithelium explain that the species Escherichia coli dominate the epidemiological profile with 75, $41 \%$ flowed by Klebsiella spp strains with $17.05 \%$. Our results were consistent with a recent notional study, Maghreb and European $[1,4,14,11]$.

Gram-positive bacteria have an adhesion factor, which is lipoteichoic acid, which may explain why Gram-positive cocci is represented with a rate of $10,5 \%$. Indeed, the 
authors of latest study in Madagascar reported 11,4\% [15]. However, the authors recent studies in India reported 20 to $30 \%$ of Gram-positive cocci causing UTI and similar result were reported in Nigeria with a Hight prevalence of S. aureus $[\mathbf{1 6}, \mathbf{1 7}]$. The epidemiological data of Uropathogens is quite important for countries and towns with poor access to healthcare services and commonly used empirical antibiotic.

The routine therapy of UTIs is performed based on the use of antibiotics, and appropriate antibiotics should be selected by considering the characteristics such as the antibiotic susceptibility pattern of the causative isolate, infection type ( community-acquired or hospitalacquired infections), the patient's conditions including age, gender, history of allergy, underlying diseases, previous antibiotic consumption, taking the other drug, history of previous UTIs, site of UTI and also the pathogenic or normal flora of the causative isolate [18]. Unfortunately, the widespread and misuse of these antibiotics resulted in the increasing rate of resistance to them in the societies. There are different risk factors associated with acquiring multi-drug resistance including the use of antibiotics before causing to UTI, hospitalization for a long time, the use of medical devices such as urinary catheters, having underlying diseases as well as an old age [18].

The study of the antibiotic resistance of strains of uropathogenic Enterobacteriaceae revealed the rate of variable resistance to the antibiotics tested.

In our study, we noted that the majority of E. coli strains are resistant to Ampicillin (63.15\%), Ticarcillin (57.87\%) and Amoxicillin / clavulanic acid (38.16\%). These results are consistent with the results of various national and European studies [1, 14]. Furthermore, $16.91 \%$ of $E$. coli strains are resistant to cephalosporin, this resistance rate is comparable to the results of some authors including Hailaji et al., 2016 who reported that resistance in E. coli strains to cephalosporins was $19.8 \%$ [4].

Résistance to $\beta$-lactamins antibiotics usually is acquired due to the production of various types of $\beta$-lactamase enzyme [18]. A group of $\beta$-lactamases, called Extended Spectrum $\beta$-lactamases is produced after mutations in the ancestral enzymes bla TEM-1, bla TEM-2, and blaSHV1 , and are often transferred by plasmids [18].

This general situation of résistance to $\beta$-lactamins antibiotics is the consequence of the selection pressure due to the wide use of $\beta$-lactamins in health facilities but also through self-medication.

Quinolones and fluroquinolones are among the most common antibiotics in treatment of UTIs and their excessive use has led to an increased resistance in UTI pathogenes $[19,18]$.

The rate of resistance to quinolones is $27.16 \%$ in our study in Casablanca. $20 \%$ in that noted in El Jadida [20] and $27 \%$ in Rabat [21].

The global epidemiological situation of resistance of E. coli strain to quinolones remains variable, in fact the resistance rate is $10 \%$ in the United State [22] s and $50 \%$ in China [23].

Mutations in DNA topoisomerases II and IV, decreased uptake of the antibiotics, extrusion of antibiotics via efflux pumps, and plasmid mediated quinolones resistance genes are the major of resistance mechanisms to these antibiotics [18]. Qnr genes (qnr A, qnr B and qnrC) are the most important plasmid mediated quinolones resistance genes that cause the antibiotic resistance by inhibiting the binding of quinolones to DNA gyrase and topoisomerases [24]. Qnr genes due to locating on different integrons resultats in the disseminated resistance in Enterobacteriaceae strains [24].

With regard to Klebsiella spp strains, From the results obtained, $53.85 \%$ of the strains studied are resistant to the combination of amoxicillin and clavulanic acid and $45.26 \%$ are resistant to cephalosporins. These results are different from those found by M. Mahmoud et al., 2011 in Algeria, which report resistance rates of $70 \%$ for Amoxicillin and Clavulanic acid, and $11 \%$ for Cefotaxime. On the other hand, this observation is reported by several notional studies, Maghreb and European, including that carried out in Nouakchott city, 
Mauritania [4] and in Tunisia [25] . In addition, we recorded $41.67 \%$ resistance for Ciprofloxacin, $43.14 \%$ for Norfloxacin, and 50\% for Ofloxacin. These results consistent with to the studies by Haillaji et al., 2016 in Mauritania where approximately $36.5 \%$ of Klebsiella ssp strains were resistant to ofloxacin and $33.6 \%$ for ciprofloxacin [4].

Regarding Proteus ssp are naturally resistant to tetracycline. For other antibiotics conventionally used in urinary tract infections, the development of resistance rates over the past decade is worrying. In our study, the resistance rates of Proteus ssp strains to Ampicillin, Piperacillin and Amicillins / Clavulanic acid were higher. This finding is consistent with that found by $\mathbf{A}$.

Mahamat., 2006 et al in the town of Naim, France [26]. The significant increase in the rate resistance to AMC, Ampicillin and Piperacillin could be explained by the ability of Proteus ssp to secrete a wide variety of plasmid $\beta$-lactamases which are responsible for lower amino-penicillin activity.

In addition, $9.27 \%$ of Proteus ssp strains are resistant to cephalosporin, results close to those reported by $\mathrm{M}$. Mahmoud et al.,2010 [27]

Fortunately, quinolones retain excellent activity on Proteus ssp strains (18\% of resistant strains), this result is similar to that found by M. Mahmoud et al., 2010 in Fez, which reports a resistance rate to ciprofloxacin of $15 \%$ [27].

The resistance of Proteus spp strains to quinolones could be linked to the emergence and extension of new resistance mechanisms, including the accumulation of mutations in genes encoding DNA gyrase and topoisomerase IV, the decrease in their intracellular concentration by an increase in the activity of efflux pumps and / or decrease in membrane permeability, and expression of the Qnr gene (Quinolone resistance) [28]. Currently, Enterobacter ssp strains are beginning to take an important place as opportunistic pathogens responsible for urinary tract infections. We noted an alarming rate of resistance to quinolones, in fact $46.15 \%$ of the Enterobacter ssp strains are resistant to ciprofloxacin, 50\% resistance to ofloxacin and $36.36 \%$ resistance to Norfloxacin. Our rates are a little high compared to several studies, in fact the resistance to quinolones in national studies varies from 0 to $50 \%$ [29, $27,30]$. On the other hand, our result is similar to the studies by TOUDJI et al., 2017 in Togo where approximately $63.89 \%$ of the Enterobacter ssp strains were resistant to Ciprofloxacin, $63.64 \%$ to Ofloxacin and $63.64 \%$ to Norfloxacin [31].

In our study, we noted an alarming rate of resistance to Aminoglycosides. Indeed, $27.83 \%$ of E. coli are resistant to Tobramycin, $42.30 \%$ of Klebsiella spp strains are also resistant to the same molecule, 61.54 of Enterobacter spp strains and 27.27 of Proteus spp strains are resistant to Tobramycin and Gentamycin. Our rates are very high compared to foreign studies. Indeed, the resistance to aminoglycosides detected in Beirut city, Lebanon and Sousse city, Tunisia remains low and varies from 4 to $10 \%[25,32]$.

The mechanism of resistance to aminoglycosides is essentially the acquisition of modifying enzymes. These enzymes belong to 3 classes corresponding to phosphorylation, acetylation and nucleotidylation activities [33].

In the present study, the presence of phenotypes resistant to all aminoglycosides should be taken into account. Indeed, the current emergence of $16 \mathrm{~S}$ RNA methyltransferases conferring a high level of resistance to all aminoglycosides used in practice is a worrying phenomenon that requires epidemiological monitoring [33].

Amikacin maintains good activity against Uropathogenic Enterobacteriaceae. However, the rational use of this molecule is mandatory in order to avoid the emergence of multidrug resistant strains.

This study underlines the importance of cytobacteriological examination of urine with antibiogram before any treatment for urinary tract infections to avoid empirical and probabilistic treatments which can promote the emergence and resistance of germs to antibiotics. 
Bacterial resistance to antibiotics poses the problem of choice of antibiotic therapy. In Africa, and in particular in Morocco, the majority of patients do not have access to the laboratory and the management of infectious syndromes is probabilistic.

In addition, this resistance is the consequence of selection pressure due to the excessive prescription and sometimes abusive use of broad-spectrum antibiotics both in hospitals and in community settings (dispensing without prescription, self-medication, free sample, etc. ...), without forgetting the impact of poorly controlled food where more and more antibiotics are used in agriculture and breeding. The predominant plasmid determinism of these acquired resistances also favors their dissemination.

\section{Conclusion}

Uropathogenic Enterobacteriaceae constitute, in our city, an increasing infectious risk with high levels of resistance to antibiotics and the emergence of resistance to last resort molecules in antibacterial therapy, they can lead, in many cases to a therapeutic impasse.

This phenomenon of multi-resistance to antibiotics is a major public health problem in Morocco, worrying and alarming because of the potential risks (increased morbidity and mortality, additional economic costs and installation of highly resistant bacteria in hospital services and in the community).

Better control in terms of strict compliance with hygiene measures, isolation of carriers, the reasoned use of antibiotics and defining therapeutic and prophylactic strategies adapted to local epidemiology are the key actions to slow down their emergence and dissemination. In addition, compliance with the rules of good pharmacy practice for the delivery of drugs, the major role that city pharmacists must play as advisers in antibiotic therapy are all elements to be promoted in controlling the spread of multi-resistance in the community.

The intra- and extra-hospital spread of multidrugresistant Enterobacteriaceae in urinary tract infections requires all healthcare establishments to monitor all patients infected with these strains.

Despite everything, other studies are necessary to determine if we are not there in the presence of the emergence of multidrug resistant Enterobacteriaceae and to associate the clinical parameters, therapeutic and the confrontation with the molecular tool allowing the classification and the typing. genes implicated in these resistances.

\section{Conflicts of Interest}

Authors declare no conflict of interest.

\section{Reference}

[1] Rakotovao-Ravahatra, Zafindrasoa Domoina, Mamy Randriatsarafara Fidiniaina, Rasoanandrasana Saïda, Raverohanta Léa, et Luc Rakotovao Andriamiadana. 2017. " Phénotypes de résistance des souches d'Escherichia coli responsables d'infection urinaire au laboratoire du Centre HospitaloUniversitaire de Befelatanana Antananarivo » Pan African Medical Journal 1-10.

[2] WHO. 2014. « Antimicrobial resistance: Global report on surveillance » World Health Organization.

[3] Reza Asadi Karam, Mohammad Mehri Habibi, et Saeid Bouzari. 2019. " Urinary tract infection: Pathogenicity, antibiotic resistance and development of effective vaccines against Uropathogenic Escherichia coli.» Molecular Immunology 56-67.

[4] Hailaji, NSM, ML Ould Salem, et SM. Ghaber. 2016.« La sensibilité aux antibiotiques des bactéries uropathogènes dans la ville de Nouakchott Mauritanie. » Elsevier Masson SAS 26 : 346-352.

[5] Cohena, R, Bingena E, Grimprela E, Raymonda J, et Gendrela D. 2011. "Résistance aux antibiotiques : un nouveau tournant à ne pas manquer. " Archives de Pédiatrie $18: 359-361$.

[6] Alem, Nabil, Frikh Mohammed, Srif Abdellatif, Maleb Adil, Chadli Mariama, Sekhsokh Yassine, Louzi Lhoucin, Ibrahimi Azzedine, Lemnouer Abdelhay, et Elouennass Mostafa. 2015. « Evaluation of antimicrobial susceptibility of Escherichia coli strains isolated in Rabat University Hospital (Morocco). » BMC Res Notes 1-4.

[7] Abouddihaj Barguigua, Idrissi Hamid Rguibi, Nayme Kaoutar, et Timinouni Mohammed. 2019. " Virulence and Antibiotic Resistance Patterns in E. coli, Morocco. » EcoHealth 1-6. 
[8] CASFM. 2018. "Comité de la Société Française de Microbiologie ». https://www.sfmmicrobiologie.org/ wpcontent/uploads/2018/12/CASFMV2_SEPTEMBRE20 18.pdf.

[9] WHO. 2020. « Antimicrobial resistance. » World Health Organization.

[10] Hamzaoui, Najia El, Berguigua Aboudihaj, Nayme Kaouter, Mohamed Sbiti, Timinouni Mohammed, et Louzid Lhoussain. 2020. «Prevalence of extended-spectrum beta-lactamases in uropathogenic Enterobacteriaceae isolated from a community setting, Meknes, Morocco. » Gene Reports 1-5.

[11] Lahlou, Amine, M, Chegri, et L'Kassmi, $\mathbf{H}$. 2009. "Épidémiologie et résistance aux antibiotiques des entérobactéries isolées d'infections urinaires à l'hôpital militaire Moulay-Ismail de Meknès. » Antibiotiques 11: 90-96.

[12] Detweiler, K, D Mayers, et SG Fletcher. 2015. «Bacteruria and urinary tract infections in the elderly. " Urol Clin North Am 561-568.

[13] M Odoki, M, Aliero A Almustapha, et J Tibyangye. 2019. « Prevalence of bacterial urinary tract infections and associated factors among patients attending hospitals in Bushenyi District, Uganda. » Int J Microbiol 1-8.

[14] Sekhsokh, Y, Chadli M, El Hamzaoui, S.A. 2008. "Fréquence et sensibilité aux antibiotiques des bactéries isolées dans les urines. \E Elsevier Masson SAS 324-327.

[15] Randrianirina, F, J L Soares, J F, Ratsimba, E Carod, V Thonnier, et P Combe. 2007. «Antimicrobial resistance among uropathogenes that cause community acquired urinary tract infections in Antananarivo, Madagascar. » J Antimicrob Chemother 153-163.

[16] B. O. R. Oladeinde, M. Olley et J. Anunibe, «Urinary tract infection in rural community of Nigeria, » N Am J Med S, 75-77, 2011.

[17] Dash, M, S Padhi, I Mohanty, P Panda, et B Parida. 2013. "Antimicrobial resistance in pathogenes causing urinary tract infections in a rural community of Odisha, India.» J Fam Community Med 20-26.

[18] Reza, Mohamed Asadi karam, Mehri Habibi, et Saeid Bouzari. 2019. «Urinary tract infection: Pathogenicity, antibiotic resistance and development of effective vaccines against Uropathogenic Escherichia coli. » Moleculer immunology 56-67.

[19] Cizman, M, Orazem A, et Krizan-Hergouth V. 2001. «Correlation between increased consumption of fluoroquinolones in outpatients and resistance of Escherichia coli from urinary tract infections.» $\mathbf{J}$ Antimicrob Agents Chemother 3.
[20] Nadmi, H, Elotmani F, Talmi M, Zerouali K, Perrier-Gros-Claude J, et Timinouni M. 2010. «Profil de résistance aux antibiotiques des entérobactéries uropathogènes communautaires à El Jadida.» Med Mal Infect 5.

[21] Tagajdid, M, Boumhil L, Iken M, Adnaoui M, et Benouda A. 2010. «Étude de la résistance des souches d'Escherichia coli isolées dans les urines aux fluoroquinolones et aux céphalosporines de troisième génération.» Med Mal Infect 3.

[22] Neuhausser, M, $\mathbf{R}$ Weinstein, et Rydman. $\mathbf{R}$. 2003. «Antibiotic resistance among Gram negative bacilli in US intensive careunits : implications for fluoroquinolones use.» JAMA.

[23] Ling, T, J. Xiong, et Y. Yu. 2008. « Multicenter antimicrobial susceptibility survey of Gram-negative bacteria isolated from patients with community-acquired infections in the People's Republic of China. " Antimicrob Agents Chemother.

[24] Shahbazi, S, M. R. A Karam, M Habibi, A Talebi, et S Bouzari. 2018. «Distribution of extended spectrum B-Lactam, quinolone, and carbapenem resistance genes and genitic diversity among uropathogenic Echerichia coli isolates in Tehran, Iran. » J Glob antimicrob Res 118-125.

[25] Larabi, K, Masmoudi A, et Fendri C. 2003. « Étude bactériologique et phénotypes de résistance des germes responsables d'infections urinaires dans un CHU de Tunis : à propos de 1930 cas » Éditions scientifiques et médicales, Elsevier SAS 5.

[26] Mahamata, A, Lavigne J.-P, Bouziges N, Daurès J.-P, et Sotto A. 2006. "Antimicrobial susceptibility of Proteus mirabilis urinary tract isolates from 1999 to 2005 at Nîmes university hospital. " Pathology Biology456461.

[27] Mahmoud, M, Benseddik N, et Yahyaoui G. 2010. «Profl de résistance des entérobactéries dans les infections urinaires. » Maroc Médical 63-67.

[28] P Nordmann, P, et H Mammeri. 2007. « Résistance plasmidique aux quinolones. » Microbiologie, Antibiotiques 246-253.

[29] Ben Haj Khalifa, A, et Khedher. M. 2010. «Fréquence et résistance aux antibiotiques des bactéries uropathogènes à l'hôpital universitaire Tahar Sfar de Mahdia.» Rev Tun Infectiol 57-61.

[30] Guérin, F. 2015. « Infections caused by Enterobacter cloacae complex: Antibiotic resistance and treatment. » Journal des Anti-infectieux 1-11.

[31] TOUDJI, AG, Bouraïma DJERI, KAROU Simplice Damintoti, Ségla TIGOSSOU, Yaovi AMEYAPOH, et Comlan de SOUZA. 2017. « Prévalence des souches d'entérobactéries productrices de bêta-lactamases. » Int. J. Biol. Chem. Sci. 1165-1177. 
[32] Hamouche, E, et Sarkis. D.K. 2011. « Evolution de la sensibilité aux antibiotiques de Escherichia coli, Klebsiella pneumoniae, Pseudomonas aeruginosa et Acinetobacter baumanii dans un CHU de Beyrouthentre 2005 et 2009 » Elsevier Masson SAS 6.

[33] Nguyen, JC, et T. Lambert. 2012. «Interprétation phénotypique de l'antibiogramme vis-à-vis des aminosides.» RFL 75-77. 